\title{
Regulation of renin expression and blood pressure by vitamin $D_{3}$
}

\author{
Curt D. Sigmund
}

Departments of Internal Medicine and Physiology \& Biophysics, 3181B Medical Education and Biomedical Research Facility, Roy J. and Lucille A. Carver College of Medicine, University of Iowa, Iowa City, Iowa 52242, USA.

Phone: (319) 335-7604; Fax: (319) 353-5350; E-mail: curt-sigmund@uiowa.edu.

J. Clin. Invest. 110:155-156 (2002). doi:10.1172/JCI200216160.
The renin-angiotensin system (RAS) has long been recognized as an important regulator of blood pressure and electrolyte balance in mammals. The RAS exerts its effects through the generation and action of angiotensin II (Ang II), which has potent vasoconstrictor, antinatriuretic, and dipsogenic properties.

Ang II is generated by the serial cleavage of angiotensinogen, first by the aspartyl protease renin, and then by the carboxydipeptidase angiotensin converting enzyme (ACE). Ang II acts as both an endocrine hormone and an autocrine and paracrine effector, notably in the kidney, heart, and brain, where it can be synthesized locally. Studies of gene-targeted mice reveal that in addition to having cardiovascular effects, the RAS is required during development, in particular during the postnatal period as development of the kidney continues (1).

\section{Renin and hypertension}

Elevated expression of gene products involved in the RAS has been observed in a number of experimental and genetic models of hypertension. Consistent with the view that abnormal RAS regulation underlies at least some forms of hypertension, infusion of Ang II causes an elevation in blood pressure, and inhibitors of the RAS pathway - particreceptor blockers - are effective antihythe rate-limiting step in Ang II synthesis, considerable attention has been paid to the transcriptional regulation of the Renin gene and to processing and secretion of its product. However, despite over two decades of study, we are only now beginning to understand some of the fundamental mechanisms regulating renin expression.

Undoubtedly, the absence of good models of renin expression has slowed ularly ACE inhibitors and angiotensin pertensive agents. Because renin acts at progress in this field, but with the availability of As.4.1, a kidney-derived cell line expressing endogenous renin mRNA (2), the past two to three years have seen significant advances in our understanding of Renin gene regulation. These cells, originally isolated from a tumor in a transgenic mouse expressing an SV40 large T antigen under control of the Renin promoter (2), represent the best available in vitro model for a juxtaglomerular cell. To date, most of what has been learned regarding Renin regulatory sequences is from deletion analysis of the $5^{\prime}$ flanking region of this gene in transiently transfected As 4.1 cells.

In this issue of the JCI, Li et al. (3) describe an innovative approach to uncover a potentially important regulatory circuit controlling renin transcription. Building upon clinical observations of an inverse relationship among plasma vitamin $\mathrm{D}_{3}\left(\mathrm{VD}_{3}\right)$ levels and blood pressure and plasma renin activity (4-6), and on the observation that $\mathrm{VD}_{3}$ supplementation reduces blood pressure in hypertensive patients $(7,8), \mathrm{Li}$ and colleagues hypothesized that vitamin $\mathrm{D}_{3}$ receptor $\left(\mathrm{VDR}_{3}\right)$ may be a primary negative regulator of renin expression. They therefore examined the levels of renin mRNA in kidneys of VDR null mice and found both renin mRNA and protein were elevated. Moreover, they showed, dietary strontium, an inhibitor of $\mathrm{VD}_{3}$ synthesis, increases renal renin mRNA levels even in normal mice, whereas direct administration of $\mathrm{VD}_{3}$ has the opposite effect. Consistent with these in vivo data, stable transfection of VDR into As4.1 cells results in $\mathrm{VDD}_{3}$-dependent reduction of both endogenous renin mRNA and Renin promoter activity.

Li et al. go to considerable lengths to show that the effects of $\mathrm{VD}_{3}$ and the VDR on renin are not indirect ones, mediated by calcium metabolism or parathyroid hormone (PTH), both of which are known to independently influence renin expression $(9,10)$. The authors confirm here that $V D R^{-/-}$mice exhibit a $30 \%$ drop in blood ionized calcium and a 150-fold increase in plasma PTH, but they show that dietary intervention to normalize blood calcium and markedly reduce plasma PTH has no effect on renal renin expression in $V D R^{-/-}$mice. Moreover, they find that renin mRNA is elevated even early in life, before changes in plasma calcium or PTH occur, and that $\mathrm{Gcm}^{-/-}$mice, which lack parathyroid glands and are hypocalcemic, do not have elevated renin mRNA. Finally, Li et al. confirm that As4.1 cells, even when stably transfected with PTH receptor $\mathrm{CDNA}$, do not modulate renin expression in response to $\mathrm{PTH}$.

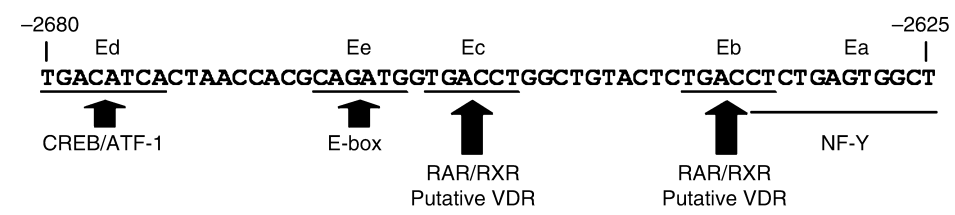

Figure 1

Schematic map of the renin enhancer. The location of binding sites (and their original designations Ea-Ee) for transcription factors is shown. Ea stands for enhancer element a and was shown to bind NF-Y. Eb and Ec (enhancer elements $b$ and $c$ ) bind to RAR/RXR and may also bind to VDR. Ed binds to the CREB/ATF family of transcription factors, while Ee binds the e-box proteins USF-1 and USF-2. 


\section{Regulation of renin transcription by $\mathrm{VD}_{3}$ and the VDR}

The VDR is a member of the thyroid hormone (T3) subfamily of nuclear hormone receptor transcription factors, which also includes retinoic acid receptor (RAR) and PPAR. All members of this family heterodimerize with retinoid $\mathrm{X}$ receptor (RXR) to form the active transactivator and recognize the cognate sequence AGGTCA-N-AGGTCA. Selectivity in receptor binding is encoded in the number of nucleotides (N) separating the AGGTCA direct repeats (11). Gross and colleagues identified an enhancer of transcription upstream of the Renin gene that is conserved in all three mouse Renin genes and the human RENIN gene (12-14). Within the enhancer lies the reverse complement to the T3R consensus sequence (TGACCT-N-TGACCT) (Figure 1), and this sequence is required for renin enhancer activity in As4.1 cells. Our group has demonstrated that 3 -tandem copies of this T3R motif (3XTRE) have the same transactivating activity as the entire enhancer, therefore bypassing the need for the binding of other transcription factors when this element is present in multiple copies (15). Interestingly, as in the current paper, we found that $\mathrm{VD}_{3}$ treatment significantly reduced activity of a transiently transfected Renin promoter-3XTRE-reporter gene in As4.1 cells (15). This clearly defines the TGACCT-N-TGACCT sequence as a potential candidate binding site for $\mathrm{VD}_{3} / \mathrm{VDR}$.

The regulation of renin transcription is very likely to be complex. The enhancer contains at least five different binding sites for transcription factors, and several of the sequences bind more than one factor (Figure 1). For instance, the putative VDR binding site is also the binding site for $\mathrm{RAR} \alpha / \mathrm{RXR} \alpha$, and retinoic acid treatment of As4.1 cells stimulates renin expression and renin promoter activity (15). The activity of the RAR/RXR binding site (and consequently the enhancer) may therefore be dependent upon the presence of either the stimulatory ligand (vitamin A) or the inhibitory ligand (vitamin D). Likewise, gel supershift and expression of dominant negative mutants of RAR clearly demonstrate that other, as yet unknown factors bind to this sequence and participate in Renin regulation.
Additional complexities are also present within the enhancer. For example, some of the binding sites are arranged to foster competition between transcription factors. The NF-Y binding site overlaps with the more promoter proximal TGACCT of the RAR response element (RARE), and the negative regulatory activity of NF-Y is dependent on this specific orientation of binding sites because NF-Y physically blocks other stimulatory factors from binding to the RARE (16) (Figure 1). Upstream of the RARE are sequences which bind members of the CREB/ATF family of transcription factors and several E-box transcription factors; mutations in either abolish enhancer activity (17).

In retrospect, given the now-known complexities associated with the "enhancer," it was probably incorrect to call the sequence an "enhancer" at all. More likely, this sequence is a compound regulatory element with numerous binding specificities and both stimulatory and inhibitory activity. Presumably, transcription factors binding to this sequence must also interact with transcription factors binding near the start site of transcription, such as the proximal promoter element, recently identified to bind HOX:PBX factors, which may help regulate expression of renin in specific cells during development (18). The importance of transcription factors that regulate renin throughout development cannot be overemphasized. Studies in Drosophila and knockout mice have illustrated the profound effects loss of Hox function can have on development. Deficiencies of both vitamin A and vitamin $D$ are likewise highly detrimental systemically $(19,20)$.

Ultimately, the transcriptional response to physiological cues probably reflects not just the activity of the VDR or other individual transactivators, but rather the interplay among the different regulators that bind the Renin enhancer and promoter. The relevance of the various binding sites must therefore be tested both in vitro and in vivo to determine which transcription factors contribute to known physiological responses. Given the hypotensive effect of $\mathrm{VD}_{3}$ and $\mathrm{VD}_{3}$-mimetics, it will also be crucial, for therapeutic diagnostic purposes, to bring this research full circle and determine the relationship between these and other transcription factors and the regulation of blood pressure.
1. Oliverio, M.I., et al. 1998. Reduced growth, abnormal kidney structure, and type 2 (AT2) angiotensin receptor-mediated blood pressure regulation in mice lacking both AT1A and AT1B receptors for angiotensin II. Proc. Natl. Acad. Sci. USA. 95:15496-15501.

2. Sigmund, C.D., et al. 1990. Isolation and characterization of renin-expressing cell lines from transgenic mice containing a renin promoter viral oncogene fusion construct. J. Biol. Chem. 265:19916-19922.

3. Li, Y.C., et al. 2002. 1,25-Dihydroxyvitamin $\mathrm{D}_{3}$ is a negative endocrine regulator of the reninangiotensin system. J. Clin. Invest. 110:229-238. doi:10.1172/JCI200215219.

4. Kristal-Boneh, E., Froom, P., Harari, G., and Ribak, J. 1997. Association of calcitriol blood pressure in normotensive men. Hypertension. 30:1289-1294.

5. Lind, L., et al. 1995. Vitamin D is related to blood pressure and other cardiovascular risk factors in middle-aged men. Am. J. Hypertens. 8:894-901.

6. Burgess, E.D., Hawkins, R.G., and Watanabe, M. 1990. Interaction of 1,25-dihydroxyvitamin D and plasma renin activity in high renin essential hypertension. Am. J. Hypertens. 3:903-905.

7. Lind, L., Wengle, B., Wide, L., and Ljunghall, S. 1989. Reduction of blood pressure during longterm treatment with active vitamin D (alphacalcidol) is dependent on plasma renin activity and calcium status. A double-blind placebo-controlled study. Am. J. Hypertens. 2:20-25.

8. Pfeifer, M., Begerow, B., Minne, H.W., Nachtigall, D., and Hansen, C. 2001. Effects of a short-term vitamin $\mathrm{D}(3)$ and calcium supplementation on blood pressure and parathyroid hormone levels in elderly women. J. Clin. Endocrinol. Metab. 86:1633-1637.

9. Antonipillai, I., and Horton, R. 1985. Role of extra- and intra-cellular calcium calmodulin on renin release from rat kidney. Endocrinology. 117:601-606.

10. Smith, J.M., Mouw, D.R., and Vander, A.J. 1983 Effect of parathyroid hormone on renin secretion. Proc. Soc. Exp. Biol. Med. 172:482-487.

11. Glass, C.K. 1994. Differential recognition of target genes by nuclear receptor monomers, dimers, and heterodimers. Endocr. Rev. 15:391-407.

12. Petrovic, N., et al. 1996. Role of proximal promoter elements in regulation of renin gene transcription. J. Biol. Chem. 271:22499-22505.

13. Shi, Q., Black, T.A., Gross, K.W., and Sigmund, C.D. 1999. Species-specific differences in positive and negative regulatory elements in the renin gene enhancer. Circ. Res. 85:479-488.

14. Yan, Y., Jones, C.A., Sigmund, C.D., Gross, K.W. and Catanzaro, D.F. 1997. Conserved enhancer elements in human mouse renin genes have different transcriptional effects in As 4.1 cells. Circ Res. 81:558-566.

15. Shi, Q., Gross, K.W., and Sigmund, C.D. 2001. Retinoic acid-mediated activation of the mouse renin enhancer. J. Biol. Chem. 276:3597-3603.

16. Shi, Q., Gross, K.W., and Sigmund, C.D. 2001 NF-Y antagonizes renin enhancer function by blocking stimulatory transcription factors. Hypertension. 38:332-336.

17. Pan, L., et al. 2001. Critical roles of a cyclic AMP responsive element and an E-box in regulation of mouse renin gene expression. J. Biol. Chem 276:45530-45538.

18. Pan, L., et al. 2001. An Abd-B class HOX.PBX recognition sequence is required for expression from the mouse Ren-1c gene. J. Biol. Chem. 276:32489-32494

19. Barrow, J.R., Stadler, H.S., and Capecchi, M.R 2000. Roles of Hoxa 1 and Hoxa 2 in patterning the early hindbrain of the mouse. Development. 127:933-944.

20. Lelievre-Pegorier, M., et al. 1998. Mild vitamin A deficiency leads to inborn nephron deficit in the rat. Kidney Int. 54:1455-1462. 